\title{
Entornos Virtuales 3D Con Juegos De Rol Y Diálogos Para Desarrollar La Comunicación Oral Del Idioma Inglés
}

\author{
Salazar Calderón Edison Hernán \\ Armas Pesántez Paul Rolando
}

Magister en Lingüística Aplicada al Aprendizaje del Ingles Docente de la Escuela Superior Politécnica de Chimborazo, Ecuador

Armas Pesántez Washington Geovanny

Magister en Lingüística Aplicada al Aprendizaje del Ingles

Docente de la Universidad Nacional de Chimborazo, Ecuador

Guadalupe Bravo Luis Oswaldo

Magister en Lingüística Aplicada al Aprendizaje del Ingles

Docente de la Escuela Superior Politécnica de Chimborazo, Ecuador

Orozco Yánez Gabriel Isaac

Ingeniero en Gestión Turística y Hotelera

Docente de la Escuela Superior Politécnica de Chimborazo, Ecuador

Allauca Peñafiel Marcelo Eduardo

Master en Interconectividad de Redes

Técnico Docente de la Escuela Superior Politécnica de Chimborazo, Ecuador

doi: 10.19044/esj.2017.v13n23p51 URL:http://dx.doi.org/10.19044/esj.2017.v13n23p51

\begin{abstract}
The present research aims at the application of a conversational English course in 3D virtual environments, to develop oral communication of the language in the students of the sixth level "G" at the Faculty of Engineering of the National University of Chimborazo, from March to August of 2014, inasmuch as, little interest in and outside the classroom was detected, which inhibited the learning process. Therefore, the use of listening and speaking skills was proposed, using communicative techniques such as role play and discussion. The development of the research was based on a quasi-experimental and qualitative design, applied type, causal, in situ and bibliographic. The method used was the hypothetical - deductive one that allowed to verify that the oral communication of the English language was developed. It was essential to develop the activities contained in the conversational course, which allowed the collection of information through evaluations with an observation sheet and qualifications scale applied to an
\end{abstract}


experimental group, represented by the sample in two scenarios: before and after applying communicative strategies; the same ones that were tabulated, analyzed, interpreted and compared, these determined that the techniques, role play and dialogue, allowed to develop the oral language skill of the students through the English course.

Keywords: Role play, Dialogues, oral expression

\section{Resumen}

La presente investigación tiene como objetivo la aplicación de un curso de inglés conversacional en entornos virtuales 3D, para desarrollar la comunicación oral del idioma en los estudiantes de sexto nivel "G" de la Facultad de Ingeniería de la Universidad Nacional de Chimborazo, durante el período marzo - agosto de 2014, pues se evidenció poco interés dentro y fuera del aula de clase, lo que inhibe el proceso del aprendizaje. Por ello se planteó el uso de las destrezas de escuchar y hablar, con técnicas comunicativas como el juego de rol y la discusión. El desarrollo de la investigación se sustentó en un diseño cuasi experimental y cualitativo, de tipo aplicado, causal, de campo y bibliográfico. El método utilizado fue el hipotético - deductivo el cual permitió comprobar que se desarrolló la comunicación oral del idioma inglés. Fue fundamental el desarrollo de las actividades contenidas en el curso conversacional, lo que permitió la recolección de la información a través de evaluaciones con una ficha de observación y escala de calificaciones aplicadas a un grupo experimental, representada por la muestra en dos escenarios: antes y después de aplicar las estrategias comunicativas; los mismos que fueron tabulados, analizados, interpretados y comparados, y a su vez determinaron que las técnicas de juegos de rol y diálogos permitieron desarrollar la expresión oral del idioma en los estudiantes a través del curso de inglés.

Palabras-claves: Juego de rol, diálogo, expresión oral

\section{Introducción}

En el Ecuador, con la Ley Orgánica de Educación Superior que rige desde 2010, en su Artículo 124 responsabiliza a las Instituciones del Sistema de Educación Superior (IES) del dominio de un idioma extranjero a ser alcanzado por parte de los estudiantes, de igual manera a través del Plan Nacional del Buen Vivir en su objetivo 4, numeral 4.8, literal i, se promueve el aprendizaje de una lengua extranjera bajo parámetros de acreditación internacional.

Pese a la obligatoriedad del dominio del idioma extranjero, en el entorno académico del Centro de Idiomas de la Facultad de Ingeniería, se 
evidencia poco interés por el aprendizaje de inglés, y a su práctica dentro y fuera del aula de clase; es decir que no se están desarrollando las habilidades lingüísticas y auditivas que le permitan el dominio exigido en el conocimiento de las estructuras gramaticales del idioma; así como también la fluidez del inglés hablado. Sin embargo, a partir de las experiencias durante el ejercicio docente y evaluación de exámenes de suficiencia del idioma inglés se logró determinar que el nivel de dominio, específicamente en la parte oral, no es el deseado.

Uno de los enfoques metodológicos considerados efectivos en el desarrollo de las destrezas lingüísticas y especialmente las de expresión oral y comprensión auditiva, es el TBA (Task-based Approach) o EBT (Enfoque basado en tareas), el mismo consta de tareas como eje del uso del lenguaje en el aula, que suministra oportunidades reales para su uso, bajo una motivación intrínseca brindando mejores contextos para promover su aprendizaje. Para la investigación se conjugo las ventajas que presenta el enfoque TBA y el uso de la tecnología, que permitió desarrollar una propuesta metodológica que constituye uno de los motivos de este trabajo de investigación, procurando contrarrestar las causas que evitan el desarrollo de la comunicación oral de los estudiantes.

Hoy en día el uso de la tecnología facilita las labores de la vida diaria y por otro lado la exigencia del conocimiento del inglés está por todas partes, por ello que aunando estas dos áreas consideradas vitales en este mundo moderno se proyectó el curso de inglés conversacional extra clase en entornos virtuales 3D, el cual constituye un novedoso aporte al campo de la enseñanza de lenguas ya que involucra el uso de tecnologías de actualidad y técnicas comunicativas motivadoras e interactivas que permitieron lograr en los estudiantes un mejor involucramiento dentro de proceso de aprendizaje y por ende el desarrollo colaborativo de su comunicación oral. En este contexto, ha surgido sin duda el encuentro con el gigantesco mundo de las Tecnologías de la Información y Comunicación (TIC) aplicadas a la educación y que paralelamente con el propósito de aprender un idioma extranjero, están constantemente en desarrollo.

Por tanto, resultó importante buscar la manera de combatir estas dificultades y atender las necesidades de los estudiantes y por ello se consideró que la aplicación del Curso de Inglés Conversacional Extra Clase en Entornos Virtuales 3D, a través de juegos de roles y discusiones, se desarrolle la comunicación oral del idioma en los estudiantes del Sexto Nivel G, de la Facultad de Ingeniería de la Universidad Nacional de Chimborazo, durante el período marzo - agosto de 2014. 


\section{Metodología}

La presente investigación es de tipo de aplicada ya que dio respuesta al problema planteado en el área educativa universitaria, a través de la búsqueda, construcción y aplicación del saber. Además, es causal, ya que se explicó las consecuencias que produce la aplicación de la propuesta en el desarrollo del proceso educativo en los estudiantes, así como también es de campo porque se realizó in-situ en la Facultad de Ingeniería de la Universidad Nacional de Chimborazo en la ciudad de Riobamba, con la finalidad de aplicar las técnicas de Juegos de Rol y Discusiones para describir, interpretar, entender la naturaleza y factores influyentes del problema. Finalmente es bibliográfica pues posee un sustento teórico para cada una de las variables de investigación.

La investigación es cuasi-experimental y para la presente investigación no se requiere de un grupo de control, pero sí admite identificar la variable independiente y las variables dependientes para poder realizar una correlación, en este caso particular se aplica el curso de inglés conversacional extra clase en entornos virtuales 3D, para luego establecer una relación entre el antes y el después del proceso de investigación con el propósito de llegar al desarrollo de la comunicación oral, a través de del estudio comparativo y analítico de los resultados obtenidos del antes y después de la aplicación de las actividades previstas en el curso de inglés.

Para la ejecución del presente trabajo de investigación se tomó como muestra el total de la población, compuesta por 18 estudiantes del sexto nivel $\mathrm{G}$ del Centro de Idiomas de la Facultad de Ingeniería de la Universidad Nacional de Chimborazo.

La investigación partió de la observación del problema y su planteamiento, para luego proceder a establecer la hipótesis general y las específicas para deducir su validez, basándose en los resultados obtenidos y procediendo con su respectiva comprobación a través de una prueba estadística que permitió determinar las conclusiones y recomendaciones.

La investigación requirió de los métodos: descriptivo, analítico sintético durante todo el proceso pues se describió los fenómenos que ocurrieron en la población y se analizó los problemas que se presentan, en pro de determinar su influencia en el ámbito educativo para el desarrollo de la comunicación oral a través del planteamiento de una hipótesis que se analizó deductiva e inductivamente.

Mediante una ficha de observación (Escala de Calificaciones), se tabuló cada uno de los parámetros, estableciendo sus frecuencias para transformarlas en porcentajes y ubicarlos en cuadros estadísticos, pasteles o barras; y posterior se compró la hipótesis por medio de la estadística Chi cuadrado. 
Se aplicó la técnica de la observación a través de una lista con escalas de frecuencias, que fueron validadas por pares académicos y que permitió la recolección de la información. La evaluación se aplicó tanto para el juego de rol compuesta por cinco parámetros; mientras que se consideraron otros parámetros para el dialogo, que permiten determinar si se cumplieron las hipótesis planteadas.

El trabajo investigativo comprende la fase teórica: que correspondió a la problematización, delimitación, objetivos, justificación, fundamentación teórica, planteamiento de hipótesis y operacionalización de las mismas. Se continuó con la fase metodológica que describió el método, tipo de investigación, población, muestra y las técnicas e instrumentos de recolección de datos. Se continuó con la fase analítica con el análisis e interpretación de resultados, conclusiones y recomendaciones.

\section{Resultados}

Mediante la ficha de observación se obtuvo los resultados del grupo experimental antes y después de aplicar la propuesta de la presente investigación. Los resultados fueron tabulados, analizados e interpretados y fueron descritos mediante una tabla, un gráfico, con su respectivo análisis e interpretación de resultados para posteriormente comparada.

La ficha de observación para la evaluación de juego de rol incluyó los siguientes parámetros: facilidad de uso de los entornos virtuales 3D; comprensión, interacción y uso adecuado de volumen y pronunciación al hablar; Uso correcto de estructuras gramaticales acordes al nivel; empleo correcto y efectivo de vocabulario y finalmente uso natural y efectivo de estrategias de conversación.

Gráfico 1: Análisis comparativo de los resultados de la aplicación de la técnica de juego de rol mediante las escalas de calificación aplicada a los estudiantes antes y después de la aplicación del curso conversacional extra clase en entornos virtuales 3D.

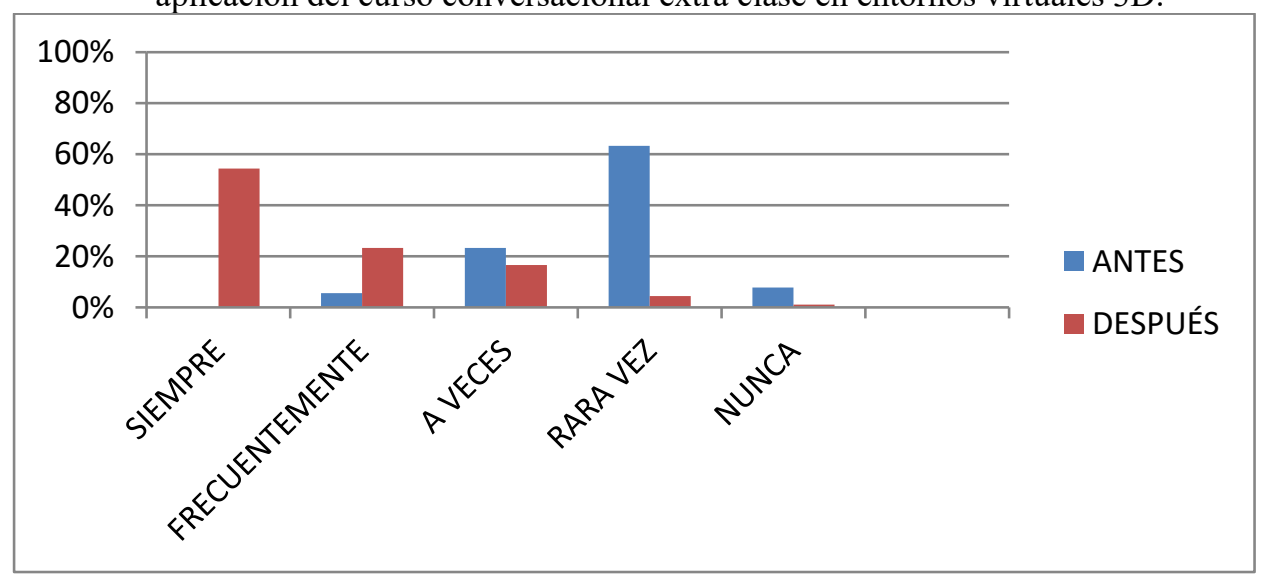

Fuente: Análisis comparativo de técnica de juego de rol

Elaborado por: Salazar Edison, 2014 
Se puede observar que antes de la aplicación del Curso Conversacional en Entornos 3D mediante la técnica del juego de rol los estudiantes tuvieron un porcentaje de $0 \%$ en el indicador de siempre, lo que da a entender que no hubo la utilización del Curso Conversacional en Entornos 3D, el indicador frecuentemente 5,5\%, a veces $23 \%$, rara vez $63.3 \%$ y nunca con un 7.7\%. Luego de la aplicación del Curso conversacional vemos que han cambiado notablemente, el indicador siempre alcanza el valor de $54.4 \%$, el de frecuentemente $23.3 \%$, a veces $16.6 \%$ y se redujeron al $4,4 \%$ y $1.1 \%$ los indicadores de rara vez y nunca respectivamente.

La ficha de observación para la evaluación de diálogos incluyó preguntas como: se expresa con adecuada velocidad, fluidez, naturalidad y confort en las palabras; es capaz de seguir gran parte de lo que se dice a su alrededor sobre tópicos generales, siempre que el lenguaje esté articulado con claridad y en un nivel estándar; es capaz de exponer un punto de vista claramente sin participar directamente en el debate; expresa creencia, opinión, acuerdo y desacuerdo cortésmente; toma parte en una discusión rutinaria sobre temas familiares que se desarrollan de manera clara $y$ articulada en un dialecto estándar y que involucra el intercambio de información verídica, recepción de instrucciones o las soluciones a problemas prácticos.

Gráfico 02: Análisis comparativo de los resultados de la aplicación de la técnica de discusión mediante la escala de calificación aplicada a los estudiantes antes y después de la aplicación del curso conversacional extra clase en entornos virtuales 3D.

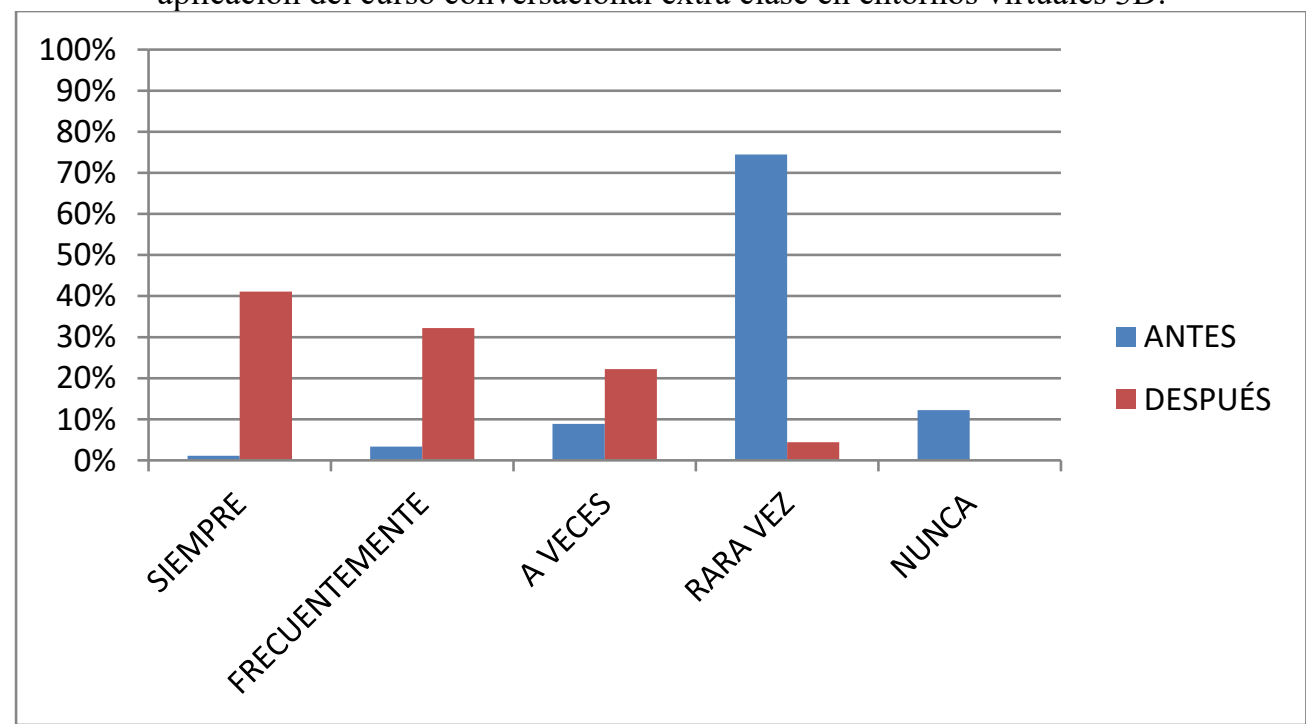

Fuente: Análisis comparativo de los resultados de la aplicación de la técnica de discusión Elaborado por: Salazar Edison, 2014 
Se puede observar que antes de la aplicación del Curso Conversacional en Entornos 3D mediante la técnica de discusión los estudiantes tuvieron un porcentaje del $1.1 \%$ en el indicador de siempre, lo que significa que no hubo la utilización de un Curso Conversacional en Entornos 3D, el indicador frecuentemente 3.3\%, a veces en $8.8 \%$, rara vez $74.4 \%$ y el $12.2 \%$ nunca. Luego de la aplicación del Curso Conversacional en Entornos 3D se ve un cambio notable en, el indicador siempre alcanza un valor del $41.1 \%$, frecuentemente alcanza el $32.2 \%$, a veces un $22.2 \%$, rara vez $4.4 \%$ y $0 \%$ en nunca.

\section{Comprobación de las hipótesis}

Paso 1: Hipótesis especifica 1

Hi: La aplicación del curso de inglés conversacional extra clase en entornos virtuales 3D, a través de juegos de roles, desarrolla la Comunicación Oral del idioma en los estudiantes del Sexto Nivel G de la Facultad de Ingeniería de la Universidad Nacional de Chimborazo, durante el período marzo - agosto de 2014.

Ho: La aplicación del curso de inglés conversacional extra clase en entornos virtuales 3D, a través de juegos de roles, no desarrolla la Comunicación Oral del idioma en los estudiantes del Sexto Nivel G de la Facultad de Ingeniería de la Universidad Nacional de Chimborazo, durante el período marzo - agosto de 2014.

Paso 2: Nivel de significación seleccionado de 0,05 . Por tanto 0,05 es la probabilidad de que se rechace una hipótesis nula.

Paso 3: Se seleccionó el estadístico chi - cuadrado que se denota por $\chi^{2}$.

Paso 4: Regla de decisión.

Columnas 5, Filas 2

$\mathrm{GL}=($ Columnas -1$)$ (Filas-1)

$\mathrm{GL}=4$ grados de libertad

$\mathrm{Gl}=4$

$\alpha=0,05$

Nivel de confianza $=95 \%$

$\chi_{t}^{2}=9.48$

En consecuencia, la regla de decisión es: $\mathrm{Si}$ el valor calculado es mayor que 9.48 se rechaza la Ho y se acepta la hipótesis de investigación Hi. Paso 5. Cálculo de Chi cuadrado. 
Tabla 01: Cálculo de Chi cuadrado - hipótesis específica 1

\begin{tabular}{|c|c|c|c|c|c|c|c|c|c|c|c|}
\hline TIEMPO DE & \multicolumn{2}{|c|}{ SIEMPRE } & \multicolumn{2}{|c|}{ FRECUENTEMENTE } & \multicolumn{2}{|c|}{ A VECES } & \multicolumn{2}{|c|}{ RARA VEZ } & \multicolumn{2}{|c|}{ NUNCA } & \multirow[t]{2}{*}{ TOTAL } \\
\hline DE LA GUÍA & $\mathrm{F}$ & $\%$ & $\mathrm{~F}$ & $\%$ & $\mathrm{f}$ & $\%$ & $\mathrm{~F}$ & $\%$ & $\mathrm{f}$ & $\%$ & \\
\hline ANTES & 0 & $0.00 \%$ & 1 & $5.55 \%$ & 4.2 & $23.33 \%$ & 11.4 & $63.33 \%$ & 1.4 & $7.77 \%$ & 18 \\
\hline DESPUÉS & 9.8 & $54.4 \%$ & 4.2 & $23.3 \%$ & 3 & $16.6 \%$ & 0.8 & $4.44 \%$ & 0.2 & $1.1 \%$ & 18 \\
\hline TOTAL & & 9.8 & & & & 7.2 & & 12.2 & & 1.6 & 36 \\
\hline
\end{tabular}

Fuente: Ficha de observación hipótesis 1

Elaborado por: Salazar Edison, 2014

Tabla 02: Contingencia - hipótesis específica 1

\begin{tabular}{|c|c|c|c|c|c|c|c|c|c|c|c|}
\hline \multirow{2}{*}{$\begin{array}{l}\text { TIEMPO DE } \\
\text { APLICACIÓN } \\
\text { DE LA GUÍA }\end{array}$} & \multicolumn{2}{|c|}{ SIEMPRE } & \multicolumn{2}{|c|}{ FRECUENTEMENTE } & \multicolumn{2}{|c|}{ A VECES } & \multicolumn{2}{|c|}{ RARA VEZ } & \multicolumn{2}{|c|}{ NUNCA } & \multirow[b]{2}{*}{ TOTAL } \\
\hline & $f_{o}$ & $f_{e}$ & $f_{o}$ & $f_{e}$ & $f_{o}$ & $f_{e}$ & $f_{o}$ & $f_{e}$ & $f_{o}$ & $f_{e}$ & \\
\hline ANTES & 0 & 4.9 & 1 & 2.6 & 4.2 & 3.6 & 11.4 & 6.1 & 1.4 & 0.8 & 18 \\
\hline DESPUÉS & 9.8 & 4.9 & 4.2 & 2.6 & 3 & 3.6 & 0.8 & 6.1 & 0.2 & 0.8 & 18 \\
\hline TOTAL & & & & & & & & & & & 36 \\
\hline
\end{tabular}

Fuente: Ficha de observación - hipótesis 1

Elaborado por: Salazar Edison, 2014

Tabla 03: Cálculo del chi-cuadrado - hipótesis específica 1

\begin{tabular}{|c|c|c|c|c|c|c|}
\hline \multirow{4}{*}{} & \multirow{2}{*}{ ALTERNATIVAS } & $f_{o}$ & $f_{e}$ & $f_{o} f_{e}$ & $\left(f_{0}-f_{e}\right)^{2}$ & $\frac{\left(f_{o}-f_{e}\right)^{2}}{f_{e}}$ \\
\hline \multirow{4}{*}{ ANTES } & Siempre & 0 & 4.9 & -4.9 & 24 & 4.8 \\
\cline { 2 - 7 } & Frecuentemente & 1 & 2.6 & -1.6 & 2.56 & 0.9 \\
\cline { 2 - 7 } & A veces & 4.2 & 3.6 & 0.6 & 0.36 & 0.1 \\
\cline { 2 - 7 } & Rara vez & 11.4 & 6.1 & 5.3 & 28 & 4.5 \\
\cline { 2 - 7 } & Nunca & 1.4 & 0.8 & 0.6 & 0.36 & 0.4 \\
\hline \multirow{4}{*}{1} & Siempre & 9.8 & 4.9 & 4.9 & 24 & 4.8 \\
\cline { 2 - 7 } & Frecuentemente & 4.2 & 2.6 & 1.6 & 2.56 & 0.9 \\
\cline { 2 - 7 } & A veces & 3 & 3.6 & -0.6 & 0.36 & 0.1 \\
\cline { 2 - 7 } & Rara vez & 0.8 & 6.1 & -5.3 & 28 & 4.5 \\
\hline \multirow{4}{*yyyyyy}{} & Nunca & 0.2 & 0.8 & -0.6 & 0.36 & 0.4 \\
\hline
\end{tabular}

Fuente: Ficha de observación - hipótesis 1

Elaborado por: Salazar Edison, 2014

El valor calculado para chi-cuadrado $(21,4)$ queda a la derecha de 9,48 y en consecuencia, la decisión es rechazar Ho al nivel 0,05. Se acepta la Hi la cual establece que: "la aplicación del curso de inglés conversacional extra clase en entornos virtuales $3 d$, a través de juegos de roles, desarrolla la comunicación oral del idioma en los estudiantes del sexto nivel g de la 
Facultad de Ingeniería de la Universidad Nacional de Chimborazo, durante el período marzo - agosto de 2014."

Zona de aceptación de la hipótesis específica $\mathrm{N}^{\mathrm{o}} 1$

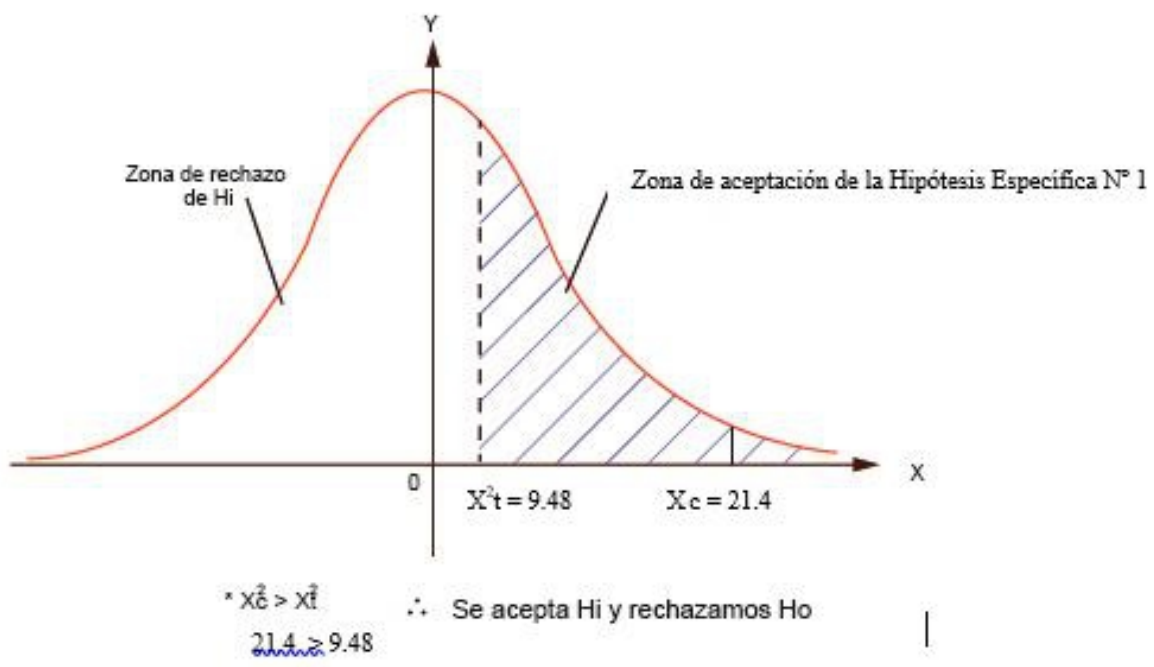

Fuente: Datos del $\chi^{2}{ }_{c}-\chi^{2}{ }_{t}$

Elaborado por: Salazar Edison, 2014

\section{Comprobación de la hipótesis específica 2}

Paso 1: Hipótesis especifica 2

Hi: La aplicación del Curso de Inglés Conversacional Extra Clase en Entornos Virtuales 3D, a través de discusiones, desarrolla la Comunicación Oral del idioma en los estudiantes del Sexto Nivel G de la Facultad de Ingeniería de la Universidad Nacional de Chimborazo, durante el período marzo - agosto de 2014.

Ho: La aplicación del Curso de Inglés Conversacional Extra Clase en Entornos Virtuales 3D, a través de discusiones, no desarrolla la Comunicación Oral del idioma en los estudiantes del Sexto Nivel G de la Facultad de Ingeniería de la Universidad Nacional de Chimborazo, durante el período marzo - agosto de 2014.

Paso 2: Nivel de significación seleccionado de 0,05 . Por tanto 0,05 es la probabilidad de que se rechace una hipótesis nula.

Paso 3: Se seleccionó el estadístico chi - cuadrado que se denota por $\chi^{2}$.

Paso 4: Regla de decisión.

Columnas 5, Filas 2

$\mathrm{GL}=($ Columnas -1$)$ (Filas- 1$)$

$\mathrm{GL}=(4)(1)$

$\mathrm{GL}=4$ grados de libertad

$\alpha=0,05$ 
Nivel de confianza $=95 \%$

$\chi_{t}^{2}=9.48$

En consecuencia, la regla de decisión es: Si el valor calculado es mayor que 9.48 se rechaza la Ho y se acepta la hipótesis de investigación Hi.

Paso 5. Cálculo de Chi cuadrado.

Tabla 04: Cálculo del Chi-cuadrado - hipótesis específica 2

\begin{tabular}{|c|c|c|c|c|c|c|c|c|c|c|c|}
\hline \multirow{2}{*}{$\begin{array}{l}\text { TIEMPO DE } \\
\text { APLICACIÓN } \\
\text { DE LA GUÍA }\end{array}$} & \multicolumn{2}{|c|}{ SIEMPRE } & \multicolumn{2}{|c|}{ FRECUENTEMENTE } & \multicolumn{2}{|c|}{ A VECES } & \multicolumn{2}{|c|}{ RARA VEZ } & \multicolumn{2}{|c|}{ NUNCA } & \multirow[t]{2}{*}{ TOTAL } \\
\hline & $\mathrm{F}$ & $\%$ & $\mathrm{~F}$ & $\%$ & $\mathrm{f}$ & $\%$ & $\mathrm{f}$ & $\%$ & $\mathrm{f}$ & $\%$ & \\
\hline ANTES & 0.2 & $1.1 \%$ & 0.6 & $3.3 \%$ & 1.6 & $8.8 \%$ & 13.4 & $74.4 \%$ & 2.2 & $12.2 \%$ & 18 \\
\hline DESPUÉS & 7.4 & 41.41 & 5.8 & $32.2 \%$ & 4 & $22.2 \%$ & 0.8 & $4.4 \%$ & 0 & $0.0 \%$ & 18 \\
\hline TOTAL & & .6 & & & & 5.6 & & 4.2 & & 2.2 & 36 \\
\hline
\end{tabular}

Fuente: Ficha de observación - hipótesis específica 2

Elaborado por: Salazar Edison, 2014

Tabla 05: Contingencia - hipótesis específica 2

\begin{tabular}{|c|c|c|c|c|c|c|c|c|c|c|c|}
\hline TIEMPO DE & \multicolumn{2}{|c|}{ SIEMPRE } & \multicolumn{2}{|c|}{ FRECUENTEMENTE } & \multicolumn{2}{|c|}{ A VECES } & \multicolumn{2}{|c|}{ RARA VEZ } & \multicolumn{2}{|c|}{ NUNCA } & \multirow[b]{2}{*}{ TOTAL } \\
\hline $\begin{array}{l}\text { APLICACIÓN } \\
\text { DE LA GUÍA }\end{array}$ & $f_{o}$ & $f_{e}$ & $f_{o}$ & $f_{e}$ & $f_{o}$ & $f_{e}$ & $f_{o}$ & $f_{e}$ & $f_{o}$ & $f_{e}$ & \\
\hline ANTES & 0.2 & 3.8 & 0.6 & 3.2 & 1.6 & 2.8 & 13.4 & 7.1 & 2.2 & 1.1 & 18 \\
\hline DESPUÉS & 7.4 & 3.8 & 5.8 & 3.2 & 4 & 2.8 & 0.8 & 7.1 & 0 & 1.1 & 18 \\
\hline TOTAL & \multicolumn{2}{|c|}{7.6} & \multicolumn{2}{|c|}{6.4} & \multicolumn{2}{|c|}{5.6} & \multicolumn{2}{|c|}{14.2} & \multicolumn{2}{|c|}{2.2} & 36 \\
\hline
\end{tabular}

Fuente: Ficha de observación - hipótesis específica 2

Elaborado por: Salazar Edison, 2014

Tabla 06: Cálculo del chi-cuadrado - hipótesis específica 2

\begin{tabular}{|c|c|c|c|c|c|c|}
\hline \multirow{4}{*}{ ALTERNATIVAS } & $f_{o}$ & $f_{e}$ & $f_{o} f_{e}$ & $\left(f_{0}-f_{e}\right)^{2}$ & $\frac{\left(f_{o}-f_{e}\right)^{2}}{f_{e}}$ \\
\hline \multirow{4}{*}{ ANTES } & Siempre & 0.2 & 3.8 & -3.6 & 12.9 & 3.3 \\
\cline { 2 - 7 } & Frecuentemente & 0.6 & 3.2 & -2.6 & 6.7 & 2 \\
\cline { 2 - 7 } & A veces & 1.6 & 2.8 & -1.2 & 1.4 & 0.5 \\
\cline { 2 - 7 } & Rara vez & 13.4 & 7.1 & 6.3 & 39.6 & 5.5 \\
\cline { 2 - 7 } & Nunca & 2.2 & 1.1 & 1.1 & 1.2 & 1 \\
\hline \multirow{3}{*}{ DESPUÉS } & Siempre & 7.4 & 3.8 & 3.6 & 12.9 & 3.3 \\
\cline { 2 - 7 } & Frecuentemente & 5.8 & 3.2 & 2.6 & 6.7 & 2 \\
\cline { 2 - 7 } & A veces & 4 & 2.8 & 1.2 & 1.4 & 0.5 \\
\cline { 2 - 7 } & Rara vez & 0.8 & 7.1 & -6.3 & 39.6 & 5.5 \\
\hline & Nunca & 0 & 1.1 & -1.1 & 1.2 & 1 \\
\hline & & & & & & $\chi_{c}^{2}=24.6$ \\
\hline
\end{tabular}

Fuente: Ficha de observación - hipótesis específica 2

Elaborado por: Salazar Edison, 2014 
El valor calculado para chi-cuadrado $(24,6)$ queda a la derecha de 9,48 y en consecuencia, la decisión es rechazar Ho al nivel 0,05. Se acepta la Hi la cual establece que: "la aplicación del curso de inglés conversacional extra clase en entornos virtuales $3 \mathrm{~d}$, a través de discusiones, desarrolla la comunicación oral del idioma en los estudiantes del sexto nivel $\mathrm{G}$ de la Facultad de Ingeniería de la Universidad Nacional de Chimborazo, durante el período marzo - agosto de 2014."

Zona de aceptación de la hipótesis específica $\mathrm{N}^{\circ} 2$

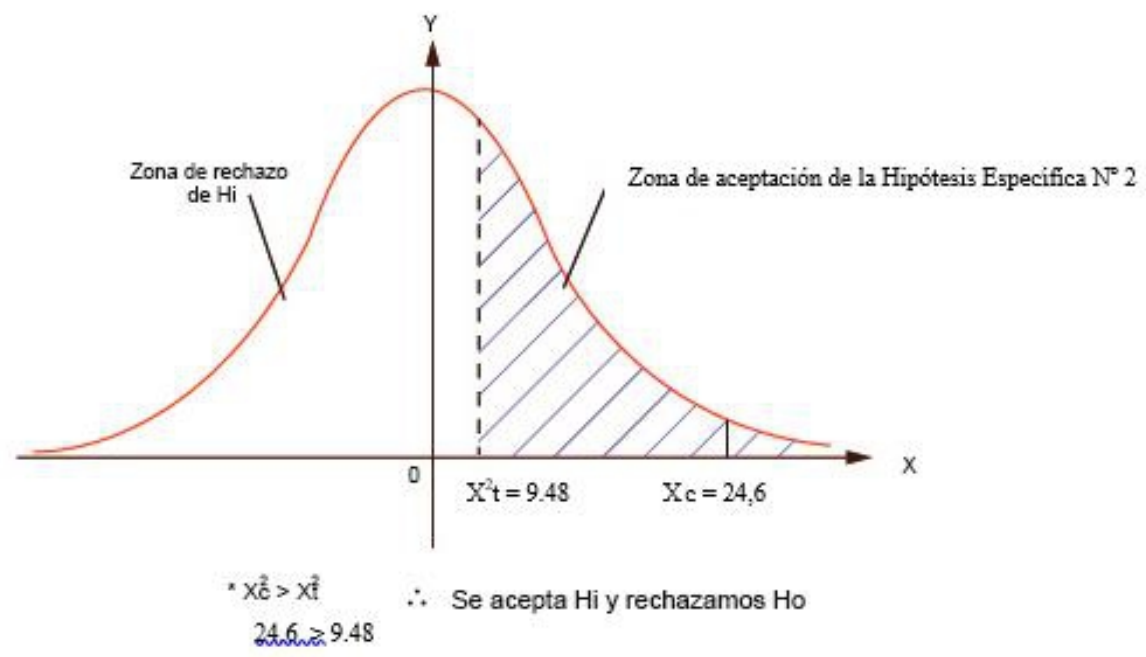

Fuente: Datos del $\chi^{2}{ }_{c}-\chi^{2}{ }_{t}$

Elaborado por: Salazar Edison, 2014

\section{Discusión}

El constructivismo plantea que nuestro mundo es un mundo humano, debido a la interacción humana con los estímulos naturales y sociales que lo hemos procesado desde nuestras operaciones mentales (Piaget, 1965). Es decir que el paradigma constructivista busca que el rol del docente se oriente correctamente, considerando que los estudiantes son los generadores del conocimiento en base a su experimentación y medio social que lo rodea.

La educación ha dado profundas transformaciones en la construcción del conocimiento, través de nuevas teorías, paradigmas y enfoques que de la mano de la tecnología han proporcionado procesos educativos especializados, que buscan lograr un conocimiento real, racional, preciso, verificable y aplicable. llamado ciencia en base a este llamado constructivismo dialéctico ya que recalca en la interacción de los individuos y su entorno (Schunk, 1997).

La perspectiva vigotskiana valora la interacción social en el aprendizaje, ya que se ha establecido que el aprendizaje no es considerado 
como una actividad individual, sino social. El aprendizaje cooperativo hace que el estudiante aprenda más eficazmente (Schunk, 1997). Por lo que es necesario, establecer el trabajo grupal y cooperativo para que la interacción social sea dinámica y motivada, mientras que se fomente la autoestima y el aprendizaje en general.

Las actividades extra clase son consideradas aquella que son complementarias a las propias de la clase y que están vinculadas o no al plan de estudios y que son dirigidas, preferentemente por los estudiantes bajo la supervisión de los docentes (Torres Y, 2012). Las actividades extra clase se convierten en una necesidad dentro de la educación integral, ya que las actividades que se desarrollan durante la clase regular a veces resultan insuficientes, y no reflejan una serie de actividades sociales, esenciales para una buena educación (Herrera, 2006).

Una guía metodológica permite sistematizar contendidos conceptuales y teóricos en base a la experiencia práctica objeto de estudio, es una forma de elaboración intelectual cuyo resultado puede expresarse en formatos diferentes, procurando hacer partícipes de los hallazgos a quienes no tuvieron la oportunidad de estar involucrados en la ejecución (Castro, 2010). En este sentido el Curso Conversacional Extra Clase en Entornos Virtuales 3D, al cumplir con estas características, no es más que una guía metodológica que contiene un número determinado de actividades de orden comunicativo y que orienta al docente en su aplicación, con el propósito de lograr los objetivos lingüísticos planteados para con los estudiantes.

Según Rahman (2010): la comunicación oral es una habilidad retórica única que es aprendida y que requiere la comprensión del qué decir y cómo decirlo. De igual forma Mikoluk (2013): manifiesta que la comunicación oral, implica el uso del lenguaje para intercambiar información con otros. Mientras que Kumar (2010): define a la comunicación oral como un intercambio del mensaje verbal entre el emisor y el receptor.

Según Fulcher (2003): enuncia que "hablar es un constructo difícil de definir" (p.2). Su mención provoca inestabilidad al poder hallar y entender el término mismo, pero se convierte en el incentivo para ir más allá y buscar evidencias que validen una acertada conciliación en torno a su definición.

Pinilla (2004) afirma que "la expresión oral es un proceso de comunicación que se desarrolla por medio de un acto comunicativo por medio del cual la información es procesada, transmitida, intercambiada y negociada entre los interlocutores". Es decir que se puede deducir que la expresión oral tiene como complemento la comprensión auditiva.

Los mundos virtuales son la evolución de los simuladores y juegos multiusuarios que aparecieron por la década de los ochenta y que tuvieron estructuras similares a las actuales e incluso fueron plataformas de interacción social. Con el acelerado desarrollo de la tecnología estos han 
venido mejorando indiscutiblemente e incorporando mayor capacidad gráfica, velocidad de procesamiento, conexión y altas especificaciones en general que el hoy en día están disponibles.

Con los datos obtenidos en la presente investigación a través del juego de roles se pudo discernir que, antes de aplicar las técnicas comunicativas, los estudiantes no habían desarrollado las habilidades básicas necesarias para el uso de la plataforma virtual 3D, lo que implico que el docente debió dotarles de información acerca de su manejo para que puedan desenvolverse adecuadamente en los entornos y realicen las actividades previstas.

También se evidenció que en un inicio la mayoría de los estudiantes no ponen en funcionamiento algunas actividades de la lengua necesarias para lograr una adecuada comunicación oral, por lo que fue necesario que los docentes realicen prácticas a través de las técnicas previstas para que desarrollen estos aspectos lingüísticos lo cual permitió que los estudiantes que sean capaces de comprender, interactuar y hacerse entender con un adecuado volumen y pronunciación gracias a que el curso conversacional contiene actividades que desarrollan estas cualidades lingüísticas .

Se pudo comprobar que en su mayoría los estudiantes aplican la gramática correctamente, y esto debido a la puesta en práctica de las estructuras gramaticales aprendidas en clases regulares, a través de las técnicas aplicadas en curso conversacional las mismas que motivan su utilización.

Se puede deducir la dificultad en los estudiantes para emplear el vocabulario correcto y efectivamente en determinada situación, pero con la estrategia comunicativa aplicadas en situaciones reales se estimuló la adquisición y puesta en práctica del mismo.

\section{Conclusion}

La elaboración del curso conversacional de inglés utilizado estrategia comunicativa a través del juego de rol y diálogo, aplicado en un entorno virtual 3D en los estudiantes del sexto nivel, permitió desarrollar planificadamente las actividades en las cuales se obtuvo las calificaciones en los escenarios del antes y después, las mismas que fueron tabuladas, analizadas, interpretadas y finalmente comparadas.

Los parámetros que se utilizaron en la lista de observación del juego de rol y diálogo permitieron evaluar individualmente cada estrategia comunicativa para determinar en conjunto el desarrollo de la expresión oral en los estudiantes; pues en el diagnóstico previo de los estudiantes, se notó una marcada falta de capacidad para dar sus puntos de vista sobre temas generales por lo se necesitó mejorar su nivel de pensamiento crítico y su 
nivel lingüístico, donde intervinieron las estrategias a través de las actividades.

La aplicación del curso conversacional extra clase en entornos virtuales 3D, a través de los juegos de rol y diálogo, desarrolló la comunicación oral de los estudiantes del Sexto Nivel G del Centro de Idiomas de la Facultad de Ingeniería, puesto que el rol registro un chicuadrado de 21,4 mientras que los diálogos registraron un chi-cuadrado 24,6, ambos valores a la derecha de la zona de rechazo que representan 9,48; por lo que después de haber aplicado el curso conversacional con sus tareas, se apreció una conversación de natural y efectiva en su interacción comunicativa y que la gran mayoría de los estudiantes pudieron expresan de forma más comprensiva. Por tanto la utilización de técnicas motivadoras y colaborativas hizo que la práctica y la interacción comunicativa sea más dinámica y divertida.

\section{References:}

1. Fulcher, G. (2003). Testing Second Language Speaking. Teaching English as a Second or

2. Kumar, R. (2010). Basic bussines communication. New Delhi: Excel Books.

3. Mikoluk, K. (2013, 15 de julio). Types of Communication: Verbal, Non-verbal and Written [Web log post]. Recuperado de https://www.udemy.com/blog/types-of-communication/.

4. Piaget, J. (1965). El lenguaje y el pensamiento. Buenos Aires: CH.

5. Pinilla, R. (2004). La expresión oral. Vademécum para la formación de profesores: enseñanza español como segunda lengua extranjera. Madrid: Editorial SGEL.

6. Rahman, M. M. (2010). Teaching Oral Communication Skills: A Task-based Approach. ESP World, 1 (27), 1-11. Recuperado de http://www.esp-world.info/Articles_27/Paper.pdf.

7. Schunk, D. (1997). Teorías del Aprendizaje. México: Atlacomulco.

8. Torres, Y. (2012). Proceso didáctico en el tratamiento de inglés. México.

9. Willis, D. \& Willis, J. (2007). Doing Task-Based Teaching. Oxford: Oxford University Press.

10. Willis, J. (1996). A framework for Task-Based Learning. Birmingham: Longman. 